Muro de la Investigación, 2020(2): julio-diciembre

ISSN:2523-2886

Doi: https://doi.org/10.17162/rmi.v5i2.1325

\title{
Rendimiento académico: universo muy complejo para el quehacer pedagógico
}

\section{Academic performance: a very complex universe for pedagogical work}

\author{
Ingrid Tacilla Cardenas ${ }^{1 a}$, Salomón Vásquez Villanueva ${ }^{2}$,
} Emilyn Elizabeth Verde Avalos ${ }^{3}$, Eloy Colque Díaz

ORCID iD: https://orcid.org/0000-0002-4606-9121 ${ }^{1}$

ORCID iD: https $/ /$ orcid.org/0000-0001-8824-6176

ORCID iD: https://orcid.org/0000-0003-2093-02083

ORCID iD: https://orcid.org/0000-0003-4555-06744

Universidad Peruana Unión, Perú ${ }^{1234}$

Recibido: 10 de enero de 2020

Aceptado: 07 de junio de 2020

\begin{abstract}
Resumen
Este trabajo tiene el objetivo de analizar los factores que inciden sobre el rendimie nto académico de los estudiantes. En la actualidad, los estudios recientes muestran la problemática relacionada con este objeto de estudio; entre otros problemas, se destaca la metodología tradicional, los factores sociofamiliares, la falta de motivación y la automotivación. Por ello, este estudio enfatiza la definición del rendimiento académico: un conjunto de notas; el avance académico responde a diferentes factores de carácter biológico, psicológico, económico y sociológico. Por lo tanto, el rendimiento académico es un constructo básico, complejo y multidimensional en el proceso de enseñanzaaprendizaje, porque identifica el avance académico del estudiante y emite un juicio de valor. En conclusión, las evaluaciones, la metodología, los factores y el rol del docente están asociados directamente con el avance académico del estudiante.
\end{abstract}

Palabras clave: rendimiento académico, pedagogía, educación, metodología de la educación

\begin{abstract}
This paper has the objective of analyzing the factors that affect the academic performance of students. Currently, recent studies show the problems related to this object of study; Among other problems, traditional methodology, socio-family factors, lack of motivation and self-motivation stand out. For this reason, this study emphasizes the definition of academic performance: a set of notes; Academic progress responds to different factors of a biological, psychological, economic and sociological nature. Therefore, academic performance is a basic, complex and multidimensional construct in the teaching-learning process, because it identifies the student's academic progress and makes a value judgment.
\end{abstract}

${ }^{1}$ Correspondencia al autor: salomonv@upeu.edu.pe 
In conclusion, the evaluations, the methodology, the factors and the teacher's role are directly associated with the student's academic progress.

Keywords: academic performance, pedagogy, education, educational methodology

\section{Introducción}

El rendimiento académico es el resultado de varios factores: biológico, psicológico, económico y sociológico; estos inciden y determinan los resultados del proceso enseñanza/aprendizaje (Estrada, 2018). Es también un indicador del aprendizaje logrado por el estudiante, por eso el sistema educativo lo toma de referencia de la calidad educativa (Colonio, 2017).

En la actualidad, los estudios muestran la problemática encontrada sobre el rendimiento académico; para muchos investigadores, este comprende las notas obtenidas durante la evaluación académica, al margen de la interacción didáctica y la pedagógica del docente. Para Navarro (2003), el rendimiento es complejo, denominado aptitud escolar o desempeño académico; las diferencias son de carácter conceptual y semántico. El bajo o alto rendimiento constituye el índice académico, la interconexión, determinados por factores socio familiares, que incrementan el riesgo de vulnerabilidad (Rodríguez y Guzmán, 2019).

Por otro lado, el bajo rendimiento académico puede originarse por diversas razones: una insuficiente metodología de enseñanza empleada por el profesor, la falta de planificación y coordinación a la hora de encargar los trabajos de investigación, problemas personales del estudiante y la situación de su entorno familiar (Colonio, 2017). De igual modo, el bajo rendimiento debe su recurrencia a inadecuados programas de estudio, cuyo carácter es tradicional; la masificación de las aulas, falta de recursos de las instituciones, el papel ausente de los padres y su actitud de creer que su responsabilidad acaba donde empieza la de los maestros (Navarro, 2003).

En el plano docente, se mantiene una pedagogía tradicional; esto produce conmoción en el ámbito educativo pues los maestros creen tener siempre la razón y quieren imponer contenidos a los estudiantes, adoptando una actitud conformista, sin una preocupación por la enseñanza; se genera apatía y desmotivación para el estudio (Estrada, 2018).

La falta de motivación del docente en el proceso enseñanza-aprendizaje, la desidia de los padres de familia y la falta de automotivación del estudiante, ocasionan lamentables resultados académicos. Se puede tener una buena capacidad intelectual y buena aptitud y a la vez un rendimiento inadecuado, muy lamentable. Muchas veces esto se debe a la 
desmotivación, la falta de interés, poco estudio, y carente de método, problemas personales, entre otros (Colonio, 2017).

Por otro lado, según los estudios de PISA (2012), el Perú aún continúa en un nivel de desempeño muy bajo a nivel internacional, si lo comparamos con la mayoría de países latinoamericanos; es uno de los países sudamericanos con bajo rendimiento académico en las áreas de matemática, lectura y ciencias, generando suma preocupación para las autoridades responsables de la conducción del país y de la educación nacional.

En consecuencia, el presente estudio es necesario, pues genera y crea estrategias metodológicas pertinentes para la adquisición de conocimiento científico y tecnológico; además, pretende estimular el desarrollo de aptitudes, valores y formación de un pensamiento crítico e independiente. De igual modo, es necesario orientar a los docentes,

padres de familia y administradores educativos, en el fortalecimiento del desarrollo académico del estudiante.

Asimismo, este estudio se considera importante en el sentido que enfatiza y subraya el verdadero sentido del rendimiento académico: constituye un constructo que puede ser estimado en cierta medida por las calificaciones obtenidas por los estudiantes (Bolaños, 2018). Asimismo, es importante abordar este tema considerando los factores importantes de carácter exógeno y endógeno, entre otros.

Para concretizar, el objetivo se aborda en diversas dimensiones del rendimie nto académico: sus definiciones, evaluación y contexto (rol del docente, metodología docente y el rol del estudiante). En tal sentido, el objetivo de este trabajo es analizar los factores que inciden sobre el rendimiento académico de los estudiantes.

\section{El rendimiento académico: conceptos}

Los teóricos no se han puesto de acuerdo en cuanto a conceptuar o explicar el constructo rendimiento académico; por ejemplo, Gonz (2009) y Willcox (2007) lo definen como el cumplimiento de las metas, logros u objetivos establecidos en el área. Para Manchego (2017), es el desarrollo del aprendizaje de las competencias en determinadas situaciones frente a diversos estímulos educativos. Por su parte, Bolaños (2018) lo define como el promedio de las notas finales y el porcentaje de aprobación de un curso o grupo de cursos. Cruz Núñez y Quiñones Urquijo, (2012) lo precisan como un indicador y medida del nivel de aprendizaje alcanzado en el aula por el estudiante, constituye el objetivo central de la educación. 
La mayoría concuerda que el rendimiento académico es el resultado del aprendizaje, producto de la interacción didáctica y pedagógica entre el docente y estudiante. Estos resultados se logran durante un periodo académico determinado, en el cual se evalúa-de manera cualitativa y cuantitativa—si se lograron los objetivos propuestos (Estrada, 2018; Martinez y Perez, 2009).

En este sentido, Bolaños (2018) confirma la existencia de numerosos estudios académicos que abordan el tema del rendimiento académico, a fin de determinar las causas que lo afectan; otros se preocupan por su definición y métodos; finalmente, están los trabajos que describen el rendimiento académico de manera numérica mediante el uso de índices y estadísticas. Por lo visto, el rendimiento académico constituye un universo muy complejo para el quehacer pedagógico.

\section{Dimensiones del rendimiento académico}

Montes y Lerner (2010) consideran las siguientes dimensiones:

\section{Dimensión académica}

Es el proceso del desarrollo académico y formativo del estudiante. Está relacionado con las variables que afectan directamente la obtención del resultado del proceso; los resultados académicos se evidencian cuantitativamente. Las notas que se obtendrán serán consideradas predictoras del buen desempeño de los estudiantes en el proceso de profesionalización.

\section{Dimensión económica}

Esta se relaciona con las condiciones que tienen los estudiantes para satisfacer sus necesidades mientras estudian: vivienda, alimentación, vestido, transporte, material de estudio, gastos en actividades de esparcimiento, entre otros. Si estas son favorables, los resultados serán satisfactorios (Tonconi, 2010).

\section{Dimensión familiar}

Comprende el contexto familiar; si favorece o limita su potencial humano y social. En el hogar se forma las bases sólidas del estudiante; en el futuro se consolida la actitud frente al estudio y al éxito académico. Es aquí (en el entorno del hogar) donde se generan patrones de comportamiento, valores y sistemas de relación entre sus miembros; esta dimensión articula integralmente el proceso de enseñanza-aprendizaje de forma significativa (Barrios Gaxiola \& Frías Armenta, 2016).

\section{Determinantes del rendimiento académico}

Manchego (2017) considera dos puntos claves: 
Poseer competencias, capacidades, destrezas y habilidades. En la construcción de su aprendizaje, el estudiante debe poseer competencias, capacidades, destrezas y habilidades de tipo cognitivo; estos se convierten en factores determinantes del rendimiento académico.

Poseer motivación académica. Los procesos motivacionales durante el proceso educativo permiten establecer aprendizajes significativos en su rendimiento académico. La motivación del docente se manifiesta durante el proceso de planificación, concentración en los aprendizajes, metacognición, investigación, retroalimentación, elogio y satisfacción por el logro, sin ansiedad o temor al fracaso (Johnson y Johnson, 1985 en Navarro, 1997).

Un estilo de aprendizaje asociado al tipo de inteligencia, la conciencia de déficit asociada a capacidad de abstracción y la motivación de la familia predicen niveles más altos de rendimiento académico (Vergel-Ortega, Martínez-Lozano, y Zafra-Tristancho, 2016).

\section{Factores del rendimiento académico}

Existen muchos factores que inciden sobre el rendimiento académico de los estudiantes. Moreira (2009) menciona que ello comprende un constructo complejo, cuyos factores son endógenos y exógenos.

\section{Factores endógenos}

Comprenden los perfiles demográficos, las condiciones familiares, antecedentes académicos y la disposición hacia un área académica (Moreira, 2009). Son las características del docente, estrategias metodológicas, relaciones socio-dinámicas. Un buen rendimiento académico depende de la motivación (superación personal) y el afecto: autorregulación (Morales \& Sepúlveda, 2015). En otras palabras, la motivación, la valoración, así como las actitudes (predisposición) y las aptitudes (habilidades cognitivas) para el estudio, son factores relevantes y asociados al rendimiento académico. Los docentes que incentivan constantemente el trabajo académico tendrán buenos resultados de aprendizaje. Este depende de la perseverancia y motivación del estudiante, concebida como una mezcla de capital cultural heredado familiarmente y con el incentivo, que el docente adopta durante los procesos de enseñanza y aprendizaje (Cervini, 2003 en Moreira, 2009).

\section{Factores exógenos}


Estos se relacionan con la variable familia, social y económico de los estudiantes, repercutiendo sobre los resultados del rendimiento académico (Manchego, 2017). Los estudiantes relacionan el buen rendimiento con la práctica pedagógica, con la presencia de un adulto significativo, con el nivel educativo y las condiciones laborales de los padres (Morales y Sepúlveda, 2015).

\section{Otros factores}

\section{Espectativa del docente}

Las expectativas del docente en el aula producen efectos sobre los estudiantes y afectan el comportamiento. La percepción que muestra el profesor hacia él produce cambios en el autoconcepto y en su nivel de motivación hacia el aprendizaje. Según Colonio (2017), los factores que influyen sobre el rendimiento académico universitario se dividen en tres grupos: factores personales, factores socio-familiares, factores pedagógicos-didácticos.

Factores personales. Incluyen: personalidad, inteligencia, falta de motivación e interés, autoestima, trastornos emocionales $\mathrm{y}$ afectivos, trastornos derivados del desarrollo biológico, trastornos derivados del desarrollo cognitivo.

Factores sociofamiliares. Incluyen: factores socioeconómicos, factores socioculturales, factores educativos, trastornos emocionales y afectivos, trastornos derivados del desarrollo biológico.

Factores pedagógico-didácticos. Entre otros, planes inadecuados, estilos inapropiados, deficiente planificación, falta de refuerzos, desconexiones, medios y recuros deficientes, estrategias inapropiadas, objetivos imprecisos y no claros.

Factores organizativos o institucionales. Ausencia de equipos, excesivo número de alumnos por aula, inestabilidad del claustro, tipo de institución educativa y ubicación

Factores relacionados con el docente. Características personales del docente, formación inadecuada, expectativa de los profesores respecto a los estudiantes, falta de interés en la formación permanente y la actualización

\section{Evaluación del rendimiento académico}

Según Espinoza, Vilca y Pariona (2014), para la evaluación del rendimiento académico se tiene en cuenta los siguientes aspectos.

\section{Observación y diálogo}

La información se recoge con un instrumento, usando diferentes estrategias y técnicas: la observación y el diálogo formal o informal.

\section{Reflexión}


Se reflexiona sobre el avance académico y sus capacidades; se emite juicio de valor sobre la situación del estudiante; es decir, si el estudiante está por debajo, se mantiene o supera el nivel de rendimiento. Asimismo, se buscan las causas del bajo rendimiento y también de los progresos.

\section{Momentos de la evaluación por competencias (socioformativa)}

Para Hernández (2013), la evaluación de competencias tiene el desafío de determinar escenarios apropiados para desarrollar el desempeño integral, la resolución de problemas y el uso de instrumentos. Aquí aparecen los siguientes elementos:

\section{Evaluación diagnóstica}

Permite diagnosticar los dominios y el conocimiento de los estudiantes, previamente, antes del inicio del programa respectivo, según las necesidades correspondientes al aprendizaje, se usan preguntas abiertas, una veces; otras cerradas (Cruz Núñez \& Quiñones Urquijo, 2012).

\section{Evaluación formativa}

Se evalúan procesos, actividades y productos, con el propósito de determinar los problemas y las dificultades sometidos a la regulación, la reorientación y la retroalimentación. Esta evaluación permite realizar la orientación de la actividad académica (Cruz Núñez \& Quiñones Urquijo, 2012).

\section{Evaluación sumativa}

Permite determinar el conocimiento y los progresos logrados durante periodos específicos. Se valora la conducta final del estudiante para la certificación de acuerdo con el logro y cumplimiento de los objetivos propuestos. Sirve además para tomar las decisiones pertinentes, a fin de fijar la calificación respecto a los objetivos planificados.

\section{Autoevaluación y rendimiento académico}

Se anima la autoevaluación y el aprendizaje autónomo a lo largo de la vida académica, lo que permite comprobar el nivel de aprendizaje de los alumnos (Cruz Núñez \& Quiñones Urquijo, 2012). Asimismo, se hacen necesarias la autoevaluación, la evaluación de los pares (coevaluación) y la evaluación del docente en el nombre de la hetero evaluación.

\section{Contexto del rendimiento académico}

\section{Rol del docente}

Para Francis (2006), la interacción docente-estudiante es el resultado directo y determinante del rol del docente, en un ambiente de empatía, efectividad y valores 
humanos. Por su parte, Mas (2014) presenta seis competencias relacionadas con la función docente:

a) Diseñar la guía docente de acuerdo con las necesidades, el contexto y el perfil profesional, en coordinación con otros profesionales.

b) Desarrollar el proceso de enseñanza-aprendizaje propiciando oportunidades de aprendizaje, tanto individual como grupal.

c) Orientar el proceso de aprendizaje del alumno propiciando acciones que le permitan una mayor autonomía.

d) Evaluar el proceso de enseñanza-aprendizaje.

e) Contribuir activamente a la mejora de la docencia.

f) Participar activamente en la dinámica académico-organizativa de la institución.

\section{Metodología del docente}

Para Hernández (2004), la metodología es la manera cómo enseñar; el método supone un camino y una herramienta concreta que emplean los docentes para transmitir los contenidos, procedimientos y principios. Izquierdo y Alonso (2010) afirman que la enseñanza de la investigación debe constituye una propuesta didáctica colaborativa, especialmente desde la opción: investigación-acción. Por otro lado, Moreno-Pinado y Velázquez-Tejeda (2017) manifiestan que los métodos de enseñanza y las estrategias didácticas permiten el desarrollo del pensamiento crítico.

Por su parte, Perez (2011) menciona que el método está relacionado con la visión de la enseñanza del docente, se adapta a su realidad, al estilo de aprendizaje y a las necesidades de cada estudiante; en otras palabras, permite comunicar los contenidos fundamentales de una determinada materia.

Cabe resaltar que los métodos de los docentes de acuerdo con la naturaleza de la temática, son: inductivo, deductivo y analógico; el primero muestra las premisas para llegar la conclusión; el inductivo señala el logro del aprendizaje mediante la observación y la generalización. Por último, el método analógico comprende una modalidad de razonamiento, y permite determinar la conclusión mediante las premisas que establecen similitudes, analogías, diferencias (Cupama, 2019). A continuación, se presenta los métodos empleados al momento de desarrollar las actividades pedagógicas.

El método expositivo. Se caracteriza por la exposición de contenidos. El docente tiene un papel directivo y el estudiante es pasivo, se limita a escuchar. Este conocimiento es formalizado y sistemático. Las fuentes de información son indirectas, no provienen de la experiencia directa de los sujetos. 
El método interactivo. Consiste en una cierta "transacción" entre docente y estudiante, mediante el debate o diálogo, a fin de profundizar en un determinado tema. Para un buen rendimiento académico son necesarios los medios y los materiales, utilizados por el docente en la etapa escolar y universitaria. En este aspecto, Moroni (2002) urge la necesidad del mejoramiento de los medios y materiales en el desarrollo del proceso enseñanza/aprendizaje. Ocaña (2012) añade que los materiales didácticos ponen en contacto al estudiante con los contenidos; estos facilitan el proceso de revisión y modificación de los conocimientos.

\section{Clases de materiales didácticos:}

- Materiales auditivos

- Materiales de imagen fija

- Materiales gráficos

- Materiales impresos

- Materiales mixtos

- Materiales tridimensionales

- Materiales electrónicos

Rol del estudiante. El estudiante genera su propio conocimiento, se identifica por ser más interactivo, crítico, espontáneo; no es simple espectador, sino activo y decidido en la labor de aprendizaje (Martinez y Perez, 2009). Lebrija, Flores y Trejos (2010) argumentan que los alumnos desarrollan sus fortalezas y analizan. El rol del estudiante según Contreras, González y Paniagua (2015) se caracteriza por:

Ser activo de su propio aprendizaje.

Mostrar autodisciplina.

Fomentar el auto aprendizaje.

Evidenciar análisis crítico y reflexivo.

Participar en grupo de trabajo colaborativo.

Actuar, crear y construir saberes personales y sociales.

\section{Conclusión}

El rendimiento académico es un constructo básico, complejo y multidimensional, experimentado durante el proceso de enseñanza-aprendizaje. Permite identificar el avance académico del estudiante y emitir un juicio de valor. Los factores que inciden sobre el aprendizaje son endógenos: las actitudes, los esfuerzos, las motivaciones, las emociones, 
las habilidades cognitivas y las expectativas de éxito; así como algunos factores familiares. En cuanto a los factores exógenos se consideran: el rol pedagógico de los docentes, las relaciones sociales del estudiante y su entorno físico. Por otro lado, las evaluaciones, las metodologías, los factores y el rol del estudiante y docente están asociados con el rendimiento académico. Por ello, es importante generar y crear estrategias metodológicas pertinentes para la adquisición de conocimiento y el fortalecimiento del rendimiento académico del estudiante.

\section{Referencias}

Barrios-Gaxiola, M. I., y Frías-Armenta, M. (2016). Factores que influyen en el desarrollo y rendimiento escolar de los jóvenes de bachillerato. Revista Colombiana de Psicología, 25(1), 63-82. https//doi.org/10.15446/rcp.v25n1.46921

Bolaños, L. C. (2018). Análisis estadístico del rendimiento académico en los cursos profesionales de los estudiantes de Ingeniería Mecánica de la Universidad de San Carlos de Guatemala, durante los años 2010 a 2015. (Tesis ) Universidad de San Carlos de Guatemala, .

Colonio, L. A. (2017). Estilos de aprendizaje y rendimiento académico de los estudiantes de los cursos comprendidos dentro de la línea de construcción - DAC-FIC-UNI. Universidad Peruana Cayetano Heredia. Universidad Peruana Cayetano Heredia, Lima, Perú.

Contreras, P. A., González, B. M., y Paniagua, P. M. (2015). El rol del estudiante en los ambientes educativos mediados por las TIC. Revista Lasallista de Investigacion, 12(2), 132-138.

Cruz-Núñez, F., y Quiñones-Urquijo, A. (2012). Importancia de la evaluación y autoevaluación en el rendimiento académico. Zona próxima: Revista del Instituto de Estudios Superiores en Educación, Universidad del Norte, (16), 96-104.

Cupama, M. (2019). Relación entre la metodología de enseñanza con el rendimiento académico. Journal of Chemical Information and Modeling. Cesar Vallejo. https://doi.org/10.1017/CBO9781107415324.004

Dorantes, J., y Tobón, S. (2017). Instrumento de evaluación: rúbricas socioformativa. Praxis Investigativa ReDIE, 9, Nro. 17, 1-8.

Espinoza, J., Vilca, C., \& Pariona, J. (2014). El Desempeño Docente y el Rendimiento Académico en el Curso de Aritmética. (Tesis de licenciatura). Universidad Enrique Guzman y Valle, Lima, Perú. 
Estrada, A. (2018). Estilos de aprendizaje y rendimiento académico. Revista Boletín Redipe, 7(7), 218-228.

Francis, S. (2006). Hacia una caracterización del docente universitario "excelente". Revista Educación, 30(1), 31-49.

Gonz, M. (2009). Relacion de burnout y el rendimiento academico con la satizfaccion frente a los estudios. Revista panamericana de salud, pp. 98-111. https://doi.org/10.1590/S1020-49892009000200007

Henao, G. (2012). Actitudes-estilos de enseñanza: su relación con el rendimiento académico. International journal of psychological research, 5(1), 133-141.

Hernández, C. (2004). Metodologías de enseñanza y aprendizaje en altas capacidades. (Monografia inedita). Universidad de la Laguna.

Hernandez, M. (2010). Expectivas en el aula el poder del profesor. Revista de investigacion, 30-52.

Izquierdo-Alonso, M., e Izquierdo-Alonso, A. M. (2010). Enseñar a investigar: una propuesta didáctica colaborativa desde la investigación-acción. Documentación de las ciencias de la información, 33, 107-123.

Lebrija, A., Flores, R., y Trejos, M. (2010). El papel del maestro, el papel del alumno: un estudio sobre las creencias e implicaciones en la docencia de los profesores de matemáticas en Panamá. Educación matemática, 22(1): 31-55.

Manchego, J. (2017). Motivación y rendimiento académico en los estudiantes de la asignatura desarrollo de proyectos productivos de la especialidad de industrias alimentarias de la Universidad Nacional de Educación 2016. (Tesis de maestria). Universidad San Martin de Porres, Lima, Perú.

Martinez, V., y Perez, O. (2009). Fracaso escolar en la educacion secundaria. Articulo Virtual, 51, 67-85.

Mas, Ó.(2014). Las competencias investigadoras del profesor universitario: la percepción del propio protagonista, de los alumnos y de los expertos. Revista de investigacion, 18(3): 255-273.

Montes, I., y Lerner, J. (2010). Rendimiento acádemico - perspectiva cuantitativa. Revista de investigacion. EAFIT.

Morales, M., y Sepúlveda, M. (2015). ¿A qué atribuyen el alto rendimiento escolar los estudiantes de buen rendimiento escolar proveniente de liceos con altos índices de vulnerabilidad? V CLABES: Quinta Conferencia Latinoamericana Sobre El Abandono En La Educacion Superior, 1-7. 
Moreira Mora, T. E. (2009). Factores endógenos y exógenos asociados al rendimiento en matemática: un análisis multinivel. Revista Educación, 33(2), 61-80. https://doi.org/10.15517/revedu.v33i2.505

Moreno-Pinado, W. E., y Velázquez-Tejeda, M. E. (2017). Estrategia didáctica para desarrollar el pensamiento crítico. REICE. Revista iberoamericana sobre calidad, eficacia $y$ cambio en educación, 15(2), 53-73. https://doi.org/10.15366/reice2017.15.2.003

Moroni, H. (2002). Nuevos desafíos, papel que debe ser asumida por la universidad. tesis. Universidad Mayor De San Marcos.

Navarro, R. (1997). Factores asociados al rendimiento académico. Revista iberoamericana de educacion, 1(1681-5653), 1-21. https://doi.org/10.4321/S157518132005000200005

Navarro, R. E. (2003). El rendimiento académico: concepto, investigación y desarrollo. REICE - Revista Electrónica Iberoamericana Sobre Calidad, Eficacia Y Cambio En Educación, 1(2), 1-15.

Ocaña, Y. (2012). Influencia de los medios y materiales didácticos y el rendimiento académico de los alumnos de la asignatura Filosofia y etica de la Universidad Cesar Vallejo, sede Lima-Norte, periodo 2012-I. (Tesis de maestria). Universidad Cesar Vallejo, Lima, Perú.

Perez, M. (2011). Metodología docente. REDU. Revista de docencia universitaria, 9(3), 75. https $/ /$ doi.org/10.4995/redu.2011.6150

PISA. (2012). Lo que los estudiantes saben y pueden hacer: rendimiento de los estudiantes en matemáticas, lectura y ciencia (2da ed.). Argentina.

Rodríguez, D., y Guzmán, R. (2019). Rendimiento académico y factores sociofamiliares de riesgo. Perfiles educativos, 41(164), 118-134. https://doi.org/10.22201/iisue.24486167e.2019.164.58925

Tonconi, J. (2010). Factores que influyen en el rendimiento academico y la deserción de los estudiantes. Revista de educación, 2(1), 45.

Vergel-Ortega, M., Martínez-Lozano, J. y Zafra-Tristancho, S. L. (2016). Factores asociados al rendimiento académico en adultos. Revista Científica, CIDC, 25(25), 206-215. https://doi.org/10.14483/udistrital.jour.RC.2016.25.a4

Willcox, M. (2007). Factores de riesgo y protección para el rendimiento académico. Revista iberoamericana de educación, 55(1): 1-9. 
